\title{
The Magellanic Cloud Interaction: Interpretation and Constraints from Observations of Carbon Stars as "Test Particles"
}

\author{
William Kunkel \\ Las Campanas Observatory, Carnegie Institution of Washington, La \\ Serena, Chile
}

Serge Demers

Université de Montréal, Montréal, P.Q., Canada

\begin{abstract}
.
Since gravity alone acts on them, and their evolutionary age restricts sampling to "mid-life", carbon stars are examined as tracers of recent dynamical evolution of the Magellanic Clouds. Ablation processes modify the distribution of $\mathrm{HI}$, and velocity outliers reflect recent tidal interactios.
\end{abstract}

\section{Introduction}

Relying on simulations to understand the dynamical evolution of the Magellanic Clouds depends on our understanding the behavior of test particles, and when our expectations are not met, errors of interpretation may ensue. The present investigation centers on carbon stars as test particles, given their favorable properties, including the assurance that, unlike neutral hydrogen streams surrounding the Clouds, gravity alone acts on stars.

Carbon stars are luminous, and can be observed spectrographically to ( $V-$ $\left.M_{v}\right) \sim 22$ with a 2.5 -m aperture. Their evolutionary phase sets in after at least half a crossing time has elpased, and does not persist beyond six $\mathrm{Gyr}$ (Frogel, Mould, \& Blanco 1990). Their kinematic information is decoupled from recent star forming environments, nor is it masked by the oldest collapse and accretion processes that gave birth to galaxies. The equivalent width of the $\mathrm{CN}$ absorption features in raw spectra is high, good correlation peaks can be measured for signal-to-noise ratios in raw spectra as low as five. Carbon stars are abundant, readily observed in large numbers, even on the outermost periphery of these systems. And gravity alone determines their motion. Hence they serve as a benchmark against which the reliability of other diagnostics can be evaluated.

\section{The SMC}

Figure 1 shows a sky projection of the SMC combining 150 carbon stars from Kunkel et al. (1997a, 1999), and an additional 48 stars from Hatzidimitriou et al. (1997). An annulus of $2.2^{\circ}$ width and $7^{\circ}$ radius about the photocenter $(l=$ $302.8^{\circ}, b=-44.3^{\circ}$ ) samples a zone of Galactocentric radial velocities, corrected 

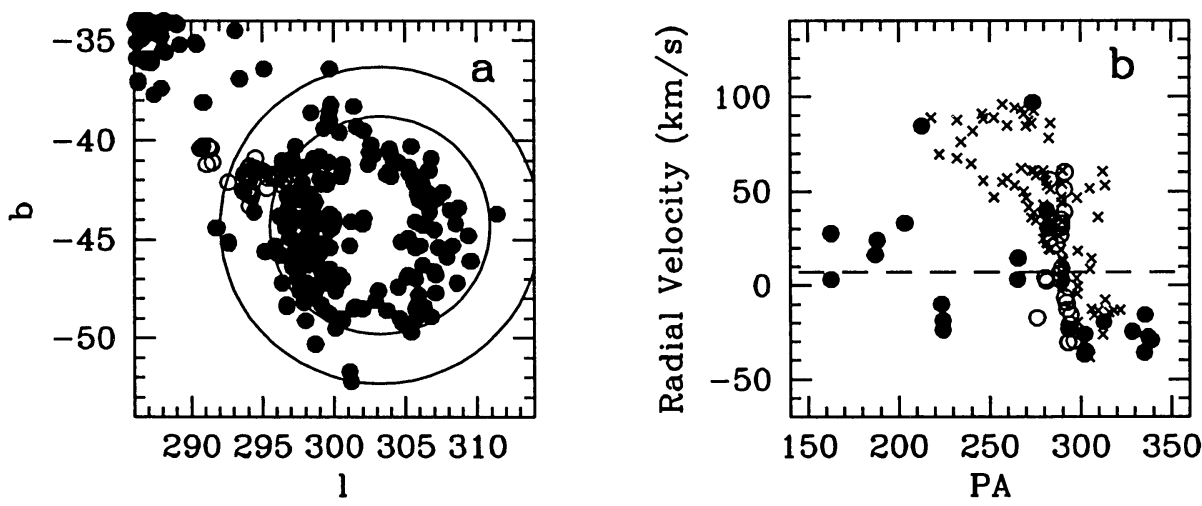

Figure 1. SMC periphery objects in Galactic coordinates, left panel. Galactocentric radial velocities vs. $P A$ within the annulus, right panel. Carbon stars are dots, early type stars are open symbols, and HI are crosses. The dashed line represents the mean SMC velocity

for motion in the plane of the sky toward $P A \sim 150^{\circ}$ of $180 \mathrm{~km} \mathrm{~s}^{-1}$ (Irwin 1996), shown in Figure 1b. If the outer portions of the SMC are currently breaking up, with an expanding velocity gradient of $8 \mathrm{~km} \mathrm{~s}^{-1} \mathrm{kpc}^{-1}$ (Hatzidimitriou et al. 1993); further, if these detached outermost components conserve their orbital angular momentum about the LMC, then a velocity difference in the line of sight of $120 \mathrm{~km} \mathrm{~s}^{-1}$ corresponds to $15 \mathrm{kpc}$ where Hatzidimitriou et al. measured, and somewhat more, closer to the LMC. In Figure $1 \mathrm{~b}$ the slope of the upper envelope of the HI velocity with $P A$ then implies that we are seeing points separated by 14 $\mathrm{kpc}$ in the line of sight, projected on but $4.4 \mathrm{kpc}$ of the sky $\left(272^{\circ}<P A<316^{\circ}\right)$ : a sheet inclined at an angle to the line of sight of $18 \pm 3^{\circ}$, with the more remote edge toward the North. Carbon stars lie predominantly at the lower velocities, approaching the observer. The HI dominates the more positive velocities and lower $P A$ 's than either the stars of early type or the majority of carbon stars. The latter lie at $P A$ 's furthest South in a continuation of the same sheet. Since the ratio of carbon stars to HI varies with $P A$, and orbital motion is to the right, or increasing $P A$ 's, the simplest hypothesis explaining the dearth of gas at larger $P A$ 's is that neutral gas on the near side has disappeared, most probably by collisional processes involving the hot Galactic corona, analogous to those observed via Fabry-Perot spectrophotometry of $\mathrm{H} \alpha$ emission on the leading edges of Magellanic Stream fragments observed by Weiner \& Williams (1996). 


\section{The LMC}

Carbon stars on the LMC periphery are seen to be asymmetrically distributed with respect to the rotation center (at $l=279.9^{\circ}, b=-33.2^{\circ}$ ), (Figure 2). In the plane of the LMC disk, inclined at $33^{\circ}$ with a line of nodes at $168^{\circ}$ (Feitzinger et al. 1977), the furthest extent of this periphery lies to the NE, near $P A \sim 55^{\circ}$, opposite the SMC, while the shortest radius lies toward the SMC. The effect was first shown in the isopleths of the LMC envelope in Figure 3 of Irwin (1991). The HI shows quite the opposite orientation (McGee \& Milton 1966). The shortest radius to the HI periphery is toward the NE in all reported surveys, including that of Putman, Gibson, and Stavely-Smith (1999).
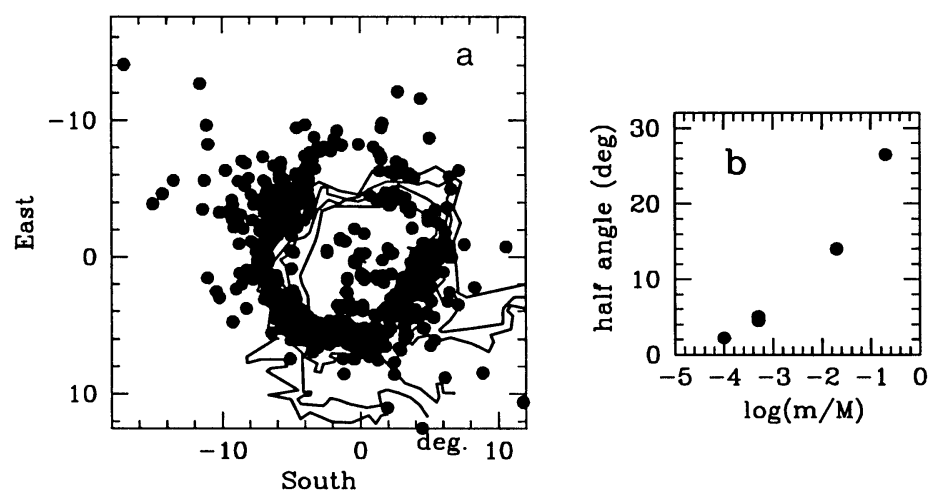

Figure 2. Map of LMC periphery carbon stars $(R A, d e c)$, with $\mathrm{HI}$ contours from McGee and Milton (1966), left panel. Half angle of debris stream vs. $\log (m / M)$, right panel.

No single mechanism can generate the dissimilarity in these asymmetries. On stars gravity alone acts, so an additional, second mechanism must act on the gas, in addition to gravity. The clue common to both the LMC and SMC is the depletion of the gas which is in the same sense in both Clouds: in the direction of orbital motion. These observations lead to the conclusion that by itself the dynamical behavior of the gas is an unreliable guide to the orbital motion of the Magellanic Clouds, obscuring the details of their origin.

\section{Closing remarks}

In earlier work Kunkel et al. (1997b) have shown the usefulness of PP $N$-body codes for interpreting the data, relying on the minimization of the parameter count as the dominant constraint. Tidal phenomena seen in those simulations were found sufficient to account for the major perturbations observed. Toomre \& Toomre (1972), Moore \& Davis (1994) and Piatek \& Prior (1995) have shown 
that a strong tidal impulse from a remote massive perturber forms a bridge and tail almost symmetrically on opposite sides of a victim. A small perturber, on the other hand, penetrating the LMC disk with pericenter toward the NE at 5 to $7 \mathrm{kpc}$ leading the LMC in orbit almost always produces an asymmetric periphery toward the NE, often leaving an off-center bar, in the manner observed (Kunkel et al. 1999).

These simulations also demonstrate a "slingshot" acceleration of a tiny fraction of LMC stars, under one percent, flung forward $15 \mathrm{kpc}$ and more, ahead in orbit, by the SMC penetration, with Galactocentric velocities between +20 and $+100 \mathrm{~km} \mathrm{~s}^{-1}$. To two carbon stars in this class from the original survey new data from Majewski et al. (1999) report six $\mathrm{K}$ giants in this category.

Besides the six "slingshots" in the six fields to the NE, the survey of Majewski et al. (1999) found some $20 \mathrm{~K}$ giants in the field NE5, all with negative velocities between -230 and $-50 \mathrm{~km} \mathrm{~s}^{-1}$, peaking near $-170 \mathrm{~km} \mathrm{~s}^{-1}$. These return to the Galaxy, appearing to belong to a population of stars in the Magellanic Plane (Kunkel and Demers 1976), whose origin in a prior encounter of the Magellanic Clouds with the Galaxy was suggested by Kunkel (1979). Their narrow distribution on the sky points to an early separation from the Magellanic progenitor. The infalling $\mathrm{K}$ giants belonged predominantly to NE5 and were not strongly evident in the adjacent fields NE4 and NE6, each some $3^{\circ}$ away. By applying equation (5) of Kunkel (1979), calibrated with a series of simulations shown in Figure 2, their distribution suggests that the mass of the tidal fragment to which they belong was less than $0.05 \%$ the mass of the Galaxy. The positive velocity "slingshot" stars, on the other hand, seen with a broader distribution covering all six NE fields, track the more energetic disruptions to which the current survivors (i.e., the MC's) of a Searle \& Zinn (1978) scenario are subjected today.

\section{References}

Feitzinger, J.V., Isserstedt, J., \& Schmidt-Kaler Th., 1997, A\&A, 57, 265

Frogel, J.A., Mould, J., \& Blanco, V.M. 1990, ApJ, 352, 96

Hatzidimitriou, D., Cannon, R.D., \& Hawkins, M.R.S. 1993, MNRAS, 261, 873.

Hatzidimitriou, D., Croke, B.F., Morgan, D.H., et al. 1997, A\&AS, 122, 507

Irwin, M.J. 1991, IAU Symp. 148, The Magellanic Clouds, eds. R. Haynes \& D. Milne (Dordrecht: Kluwer), p.453

Irwin, M.J. 1996, personal communication

Kunkel, W.E. 1979, ApJ, 228, 718

Kunkel, W.E., \& Demers, S. 1976, ROB No. 182, p.241

Kunkel, W.E., Demers, S., \& Irwin, M.J. 1997a, A\&AS, 122, 463

Kunkel, W.E., Demers, S., \& Irwin, M.J. 1997b, ApJ, 488, L129

Kunkel, W.E., Demers, S., \& Irwin, M.J. 1999, AJ, submitted

Majewski, S.R., Ostheimer, J.C., Kunkel, W.E., et al. 1999, this volume

McGee, R.X., \& Milton, J.A. 1966 Austr. J. Phys. 19, 515

Moore, B., \& Davies, M. 1994, MNRAS, 270, 209 
Piatek S., \& Prior, C. 1995, AJ, 109, 1071

Putman, M.E., Gibson, B.K., \& Staveley-Smith, L. 1999, this volume

Searle, L., \& Zinn, R. 1978, ApJ, 225, 357

Toomre, A., \& Toomre, J. 1972, ApJ, 178, 623

Weiner, B.J., \& Williams, T.B. 1996, AJ, 111, 1156

\section{Discussion}

Nicholas Suntzeff: Does your model explain the existence of the Cowley \& Harwick carbon stars in the LMC which are apparently young, blue and have negative velocities with respect to the LMC center?

Kunkel: The young carbon $\mathrm{CH}$ stars show strong negative velocity excesses to $-40 \mathrm{kms}^{-1}$ below the HI velocities, and this effect is shared by the carbon stars we observed and the LPV's. The fact that the 5 globular clusters also show this feature suggests that either the entire LMC disk responded to the SMC impact 0.2 Gyr ago, or the HI did so. No SMC debris appears to be involved in this feature. But 0.2 Gyr ago, the SW piece of the LMC lay where the SMC penetrated.

Mary Putman: Can you show me the regions which were observed on your map of the carbon star distribution and the HI contours? Do you have an idea of what mechanism is leaving the carbon stars behind (or in front of) the HI?

Kunkel: The limits for the LMC lie at $-76^{\circ}$ to the South, $-58^{\circ}$ to the North (except for SRC fields 161 and 162), and fields 55 and 58 to the West and East, respectively. For the $\mathrm{SMC}$ the radius is approximately $10^{\circ}$.

The mechanism leaving carbon stars to the East of the LMC would appear to be one of two: (1) either ram pressure is active, or (2) collisional processes ionize the hydrogen "out of sight." The presence of carbon stars on the single velocity envelope of the inter-cloud hydrogen suggests that the latter dominates. Moore \& Davis (MNRAS 1994) seem to offer the best treatment of such physics.

Mary Kontizas: Do you think that the SMC when its orbit passes by the LMC could produce an infall of the outer region's gas to the inner parts of the LMC? I have in mind the young inner LMC clusters and what effect has been responsible for their formation.

Kunkel: Toomre and Toomre (1972 ApJ) teaches us such debris should "get trapped" roughly in an inclined plane through the LMC disk. But I cannot suggest where to look for these. If we could find reliable spectral signatures identifying the origin of a cluster ( $r$-to-s process ratio?) I should not be surprised if clusters such as NGC 1818 were "acquired" by the LMC that way. 